Proc. 13th International School on Theoretical Physics: Symmetry and Structural Properties of Condensed Matter

\title{
The AC Conductivity and the Jonscher Exponent in the One-Dimensional Model of Proton Conductivity
}

\author{
T. MASŁOWSKI* \\ Faculty of Mathematics and Applied Physics, Rzeszów University of Technology, \\ al. Powstańców Warszawy 8, 35-959 Rzeszów, Poland
}

\begin{abstract}
The one-dimensional chain of rods equipped with the Grotthuss mechanism is considered as the model of proton diffusion. The AC protonic conductivity is calculated and the Jonscher exponent is found. It is established that the nature of conduction is of purely Debye's type.
\end{abstract}

DOI: 10.12693/APhysPolA.135.1263

PACS/topics: proton conductors, kinetic Monte Carlo, Jonscher exponent

\section{Introduction}

The kinetic Monte Carlo (KMC) method has proven its predictive power in applications to the dc conductivity of proton conductors [1-3]. Models adopting KMC effectively take into account a diverse spectrum of factors responsible for proton migration like the shape of hydrogen bond potential, lattice vibration, or thermal expansion of the medium and thus may support experiments with the proper interpretation of the data as well as a prediction of new phenomena. In spite of its benefits, very few applications of KMC to AC conductivity may be found in the literature [4]. The reason is: a straightforward calculation of time evolution in the case of sinusoidally varying external electric field applied to a sample of proton conductor consumes a lot of CPU time. Moreover, as the desirable quantity describing the electrical properties of the system is conductivity as a function of frequency, $\sigma(\omega)$, one needs to run the KMC calculation many times to get the result. A possible solution to this difficulty is the use of the Kubo linear response theory which connects $\sigma(\omega)$ with the diffusive properties of the system. In this paper we want to present difficulties which one has to overcome to effectively benefit from the Kubo formula doing the KMC calculation. The response function is calculated in the model previously studied, where a free flow of protons is limited only by the Coulomb interaction which acts on protons occupying the minima of the same hydrogen bond.

\section{AC conductivity}

The model consists of one-dimensional chain of rotating rods with ends occupied by protons. Protons can also migrate by hopping from one rod to the nearest one and the open boundary conditions are imposed. The physical analogues are heterocyclic based polymers. Schematically the model is presented in Fig. 1.

\footnotetext{
*e-mail: tmaslowski@prz.edu.pl
}

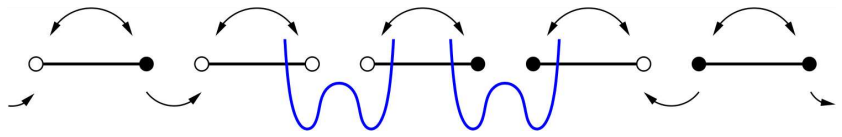

Fig. 1. Protons (solid dots) can hop to the neighbour rod if there is a vacancy (empty dot). Rods with or without protons can rotate. Rotations and hoppings are provided by the Grotthuss mechanism. If there is already a proton in one minimum of the hydrogen bond (wavy line) then it is energetically unfavourable for another proton to go to the second minimum.

The Kubo formula for the electric susceptibility in the case of discrete $\mathrm{KMC}$ movements reads as

$$
\begin{aligned}
& \chi(t)=\frac{\theta(t)}{k_{\mathrm{B}} T} C(t), \\
& C(t)=\left.\lim _{\Delta t \rightarrow \infty} \frac{1}{L^{2} \Delta t} \sum_{k \geq l} \Delta x_{k} \Delta x_{l}\right|_{t=(k-l) \delta t},
\end{aligned}
$$

where $\theta(t)$ is the Heaviside step function, $C(t)$ is the autocorrelation function of the microscopic protonic current, $L$ is the chain's length and $\Delta x_{k}$ is a change of proton position in the $k$ movement. In the KMC simulation the time evolution is discrete with time steps which are not constant but drawn in every step. Thus, the condition $t=(k-l) \delta t$ in $(2)$ has to be understood that for $t \in[(k-l-1) \delta t,(k-l) \delta t]$ the time $t$ is set to $(k-l) \delta t$, where $\delta t$ is the average KMC time step.

The Fourier transform of $\chi(t)$,

$$
\chi(\omega)=\int_{0}^{\infty} \chi(t) \mathrm{e}^{\mathrm{i} \omega t} \mathrm{~d} t,
$$

can be easily related to the conductivity

$$
\begin{aligned}
& \chi(\omega)=\chi^{\prime}(\omega)-\mathrm{i} \chi^{\prime \prime}(\omega), \\
& \sigma(\omega)=\sigma^{\prime}(\omega)+\mathrm{i} \sigma^{\prime \prime}(\omega), \\
& \sigma^{\prime}(\omega)=\varepsilon_{0} \omega \chi^{\prime \prime}(\omega), \\
& \sigma^{\prime \prime}(\omega)=\varepsilon_{0} \omega\left(1+\chi^{\prime}(\omega)\right),
\end{aligned}
$$


When using the Kubo formula there are two critical features governing the quality of $\chi(\omega)$. The first is the autocorrelation function. The KMC calculation introduces a time scale equal to the average time granulation which is governed by the system properties, i.e. the system size. Going below this threshold is possible but requires more extensive calculation. Thus, it is natural to adopt this time scale as the minimal time step. This means that our knowledge of $C(t)$ is incomplete and $\chi(\omega)$ can only be approximated. The second problem is a proper calculation of the Fourier transform. The difficulties here arise from the high frequencies which implies a highly oscillating function to be integrated. A straightforward application of a discretization costs a lot of calculational time to get a desire accuracy. Other procedures of dealing with highly oscillating integrals like Filon [5] is not improving the calculation time noticeably. More effective methods like Levin method [6] cannot be used as they need the analytical expression of the integrand. Some authors [4] are trying to avoid the problem with the accuracy of the Fourier transform by using fits to the behaviour of equivalent circuit model, but this requires in advance the knowledge about the nature of protonic current in the analysed material. It was found that a very effective way of proceeding is the use of the Gauss-Laguerre quadratures. Within this method one needs two to three orders of magnitude less number of points than in cases mentioned above. The abscissas and weights for $N$-point Gauss-Laguerre quadrature with large $N, 10^{3}$ and more, can be calculated for example within Mathematica programme with a great precision.

The efficient way of calculating the Fourier transform (3) is especially important when the interest is focused on high frequency behaviour of $\sigma(\omega)$. The Jonscher power law [7] conjectures that $\sigma(\omega)$ behaves like $\omega^{n}$ in a wide range of accordingly large frequencies. Thus, to calculate the Jonscher exponent $n$ a proper dealing with high frequency is indispensable.

Figure 2 shows the result of KMC calculations for $\chi^{\prime}(\omega)$ and $\chi^{\prime \prime}(\omega)$. Figure 2 was obtained for the value of the Coulomb interaction, $V_{\text {Coul }}$, between protons when they are at minima of the same hydrogen bond [1] equal to $0.05 \mathrm{eV}$, but qualitatively the same picture is obtained for other values $(0-0.2 \mathrm{eV})$. According to (6) the Jonscher exponent is equal to zero $\left(1+\right.$ the slope of $\chi^{\prime \prime}(\omega)$ at large $\omega$ ).

The behaviour shown in Fig. 2 and $n=0$ is characteristic for non-interacting carriers. It is seemingly surprising that the Coulomb interaction in the form built into the model cannot be considered as the nearest neighbours interaction. To get the Jonscher exponent $n \sim 0.3-0.4$, characteristic for the nearest neighbours interaction [8], the interaction cannot be limited to isolated events in time. The Coulomb interaction in the form presented in the model is sufficient to explain the macroscopic properties [1-3] but it cannot go beyond a free evolution in the case of AC conductivity which is subjected to microscopic phenomena.

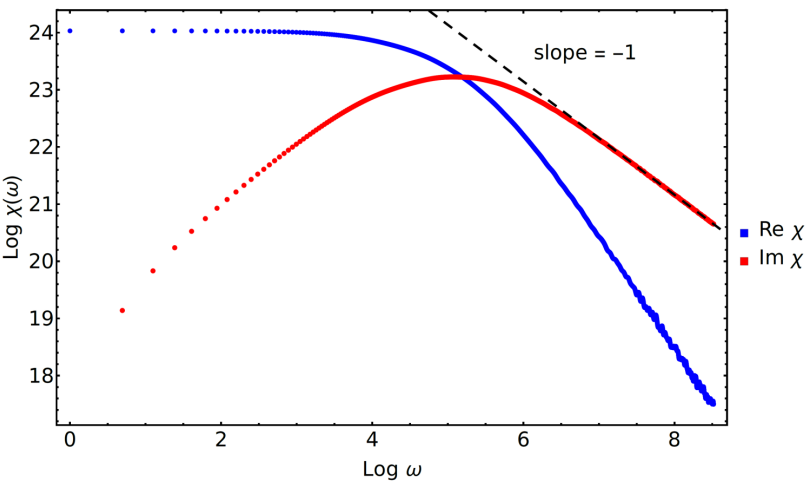

Fig. 2. The shape of $\chi(\omega)$ at $\log -\log$ scale together with the fit to $\chi^{\prime}(\omega)$ at large $\omega$. The dashed line represents the fit at large $\omega$, its slope is approximately -1 which means that the Jonscher exponent is equal to 0 . The plot was obtained for $V_{\text {Coul }}=0.05 \mathrm{eV}$ but the same behaviour were observed for all examined values of the Coulomb interaction, i.e. thought $\chi(\omega)$ is changed, the slope remains equal to -1 .

\section{Conclusions}

The AC conductivity found in the presented model reveals the characteristic behaviour for non-interacting carriers [8]. The Coulomb interaction embedded into the model plays a role of geometrical blocking rather than a nearest neighbours interaction. As the purely Debye behaviour can be found analytically, the model is a good testing ground for numerical calculations of the answer function (susceptibility) or conductivity. Although, it is possible to read out different electrical properties from the model, it has to be extended by proton interaction, e.g. by taking into account the internal polarizability, to be able to reproduce a more realistic AC behaviour and to get non-zero value of the Jonscher exponent.

\section{Acknowledgments}

I gratefully acknowledge discussions with M. Zdanowska-Frączek.

\section{References}

[1] T. Masłowski, A. Drzewiński, J. Ulner, J. Wojtkiewicz, M. Zdanowska-Frączek, K. Nordlund, A. Kuronen, Phys. Rev. E 90, 012135 (2014).

[2] T. Masłowski, A. Drzewiński, P. Ławniczak, M. Zdanowska-Frączek, J. Ulner, Solid State Ion. 278, 114 (2015).

[3] T. Masłowski, A. Drzewiński, P. Ławniczak, J. Ulner, Solid State Ion. 272, 166 (2015).

[4] E. Lee, F.B. Prinz, W. Cai, Electrochem. Commun. 12, 223 (2010).

[5] L.N.G. Filon, Proc. R. Soc. Edinburgh 49, 38 (1928).

[6] D. Levin, J. Comput. Appl. Math. 67, 95 (1996).

[7] A.K. Jonscher, Nature 267, 673 (1977).

[8] K.L. Ngai, A.K. Jonscher, C.T. White, Nature 277, 185 (1979). 